\title{
VARIABILIDADE ESPACIAL DO NÚMERO MÉDIO DE PERFILHOS E RENDIMENTO DA CULTURA DE TRIGO $\left({ }^{1}\right)$
}

\author{
MARI ROMAN $\left({ }^{2}\right)$; MIGUEL ANGEL URIBE-OPAZO $\left({ }^{3}\right)$; LÚCIA HELENA PEREIRA NÓBREGA $\left({ }^{3}\right)$; \\ JERRY ADRIANI JOHANN $\left({ }^{4}\right)$
}

\begin{abstract}
RESUMO
Este trabalho estudou a variabilidade espacial do número médio de perfilhos e rendimento da cultura do trigo, em uma área de 22,62 ha, no município de Cascavel (PR), considerando 89 pontos amostrais com distância de $50 \mathrm{~m}$ entre si. Foi realizado o levantamento do número médio de perfilhos por planta aos 30 e 60 dias após a semeadura (DAS) e o rendimento da cultura. Os dados foram analisados com técnicas geoestatísticas para estudo da estrutura de variabilidade espacial e posterior estimação de valores para locais não amostrados pelo interpolador por krigagem. Ocorreram diferenças no número de perfilhos médios aos 30 e 60 DAS, devido ao excesso de chuva em curto intervalo de tempo, provocando a morte de perfilhos após 30 DAS. Observaram-se, no número médio de perfilhos aos 30 e 60 DAS, respectivamente, fraca e forte dependência espacial. O rendimento foi de moderada dependência espacial, existindo correlação espacial entre as variáveis em estudo.
\end{abstract}

Palavras-chave: geoestatística, dependência espacial, krigagem.

\section{SPATIAL VARIABILITY OF TILLER MEAN NUMBER AND WHEAT YIELD}

\begin{abstract}
This trial aimed at studying the spatial variability of tiller mean number, as well as wheat crop yieldin 22.62 hectares, in Cascavel, Paraná State, Brazil. Eighty-nine samples points were analyzed, with a $50 \mathrm{~m}$ distance between each one. The tiller number registering was done by plant at 30 and 60 days after sow (DAS), as well as wheat crop yield. The data were recorded by geostatistics techniques in order to study spatial variability structure and future estimated values for non sampled places by kriging interpolation. There were differences on tiller number at 30 and 60 DAS due to the great amount of rain in a short term, which caused death on tillers after 30 DAS. The mean tiller number on 30 and 60 DAS showed a little spatial dependence and strong spatial dependence, respectively. The productivity presented moderate spatial dependence, since there was a spatial correlation among the considered variables.
\end{abstract}

Key words: Geostatistics, spatial dependence, kriging.

$\left({ }^{1}\right)$ Parte da dissertação de mestrado da primeira autora. Recebido para publicação em 23 de fevereiro de 2007 e aceito em 23 de novembro de 2007.

$\left(^{2}\right)$ Mestre em Engenharia Agrícola pela Universidade Estadual do Oeste do Paraná. E-mail: mari.roman19@hotmail.com

$\left({ }^{3}\right)$ Programa de Pós-graduação em Engenharia Agrícola, CCET, Universidade Estadual do Oeste do Paraná. Rua Universitária 2069, 85819-110 Cascavel-(PR). E-mail: mopazo@unioeste.br $\left({ }^{*}\right)$ autor correspondente; lhpn@unioeste.br

$\left({ }^{4}\right)$ CCET, Universidade Estadual do Oeste do Paraná. Rua Universitária , 2069, Cascavel (PR). jerryaj@pop.com.br 


\section{INTRODUÇÃO}

O conhecimento das características fisiológicas e agronômicas de uma cultura durante seu ciclo contribui no desenvolvimento de técnicas de produção. Assim, uma das características da planta de trigo são os perfilhos, os quais são unidades modulares que surgem em plantas da família gramínea (Poaceae). O perfilhamento das plantas é importante para a produção da espécie e espera-se que o maior número de perfilhos acarrete maior rendimento. $\mathrm{O}$ trigo tem por característica desenvolver muitos perfilhos, que, na maioria das vezes, não são férteis, e dessa maneira, o potencial de perfilhamento da espécie não está expresso em rendimento de grãos (TONET, 1999).

O número de unidades modulares que uma planta vai desenvolver depende de uma série de fatores, inclusive climáticos. CunHA et al. (2001), estudando Paspalum atratum cv, relataram que a disponibilidade de água do solo favorece o aumento do número de perfilhos emersos. TURNER e BEGG (1978) relataram que o encharcamento do solo pode provocar redução no número de perfilhos emersos e até mesmo sua morte.

A falta de relação direta entre número médio de perfilhos e rendimento da cultura pode ser compreendida pela relação que existe entre perfilho (broto lateral) e o eixo principal, cuja conexão vascular entre eles é um aspecto importante para o crescimento dos perfilhos, considerando que o transporte de material orgânico e inorgânico dentro da planta é realizado pelos tecidos vasculares. Segundo Alves (2000) citando Mcall (1934), interrupções nas conexões vasculares entre o eixo principal de plantas de trigo e as gemas podem inibir o desenvolvimento de gemas laterais. TAMAS (1995) sugeriu que, quando a formação da conexão vascular não promove o desenvolvimento da gema lateral, os tecidos dessa região podem não ser funcionais.

Para estudar a relação entre número médio de perfilhos e rendimento em uma área em estudo, é necessário conhecer sua ocorrência em distintos espaços e períodos, o que permite compreender o comportamento e as possíveis relações que existem entre as variáveis chamadas regionalizadas. Os mapas temáticos do número médio de perfilhos por plantas, em distintos períodos, podem auxiliar na compreensão do comportamento das variáveis regionalizadas e nas possíveis relações com o rendimento.

Matheron (1963) definiu uma variável regionalizada em uma função espacial numérica que varia de um local para outro, com continuidade aparente e cujos valores são relacionados, de algum modo, com a posição espacial que ocupam. Esse tipo de variável apresenta característica aleatória, pois os valores numéricos observados podem variar casualmente de um ponto a outro no espaço e é espacialmente correlacionada, ao mesmo tempo, variando muito no espaço, e não são inteiramente independentes. A geoestatística é um método embasado na teoria das variáveis regionalizadas e oferece técnicas para o estudo deste tipo de variável. Ela determina a estrutura de dependência espacial entre amostras e permite elaborar mapa temático de variável regionalizada por meio da interpolação por krigagem (CRESSIE, 1993).

O objetivo deste trabalho foi obter a estrutura de dependência espacial do número médio de perfilhos por planta de trigo, coletados aos 30 e 60 dias após a semeadura (DAS) comparando-os com o rendimento da cultura, por meio de mapas temáticos e a correlação espacial entre as variáveis regionalizadas a diferentes distâncias na área em estudo.

\section{MATERIAL E MÉTODOS}

Os dados foram coletados no ano agrícola de 2003, em um Latossolo Vermelho Distroférrico (EMBrapa, 1999). A área de 22,62 ha, pertencente ao Centro de Pesquisa Eloy Gomes da Cooperativa Central de Pesquisa Agrícola - COODETEC, situa-se entre coordenadas geográficas $53^{\circ} 26^{\prime} \mathrm{W}$ de longitude e $25^{\circ} 56^{\prime} \mathrm{S}$ de latitude, no município de Cascavel, Região Oeste do Estado do Paraná. Foram cultivados 18,1 ha com a cultivar COODETEC 103 (CD 103) e 3,52 ha com a cultivar COODETEC 101 (CD 101). O levantamento topográfico do solo e o posicionamento dos pontos amostrais foram realizados com auxílio de um receptor GPS da marca Trimble, modelo GeoExplorer3, com método estático de correção pósprocessada (DGPS). A malha amostral foi de $50 \mathrm{x}$ $50 \mathrm{~m}$, totalizando 89 pontos amostrais (Figura 1), correspondendo a aproximadamente quatro amostras por hectare, os quais foram demarcados com estacas e utilizados em todas as amostragens, sendo a unidade de análise um quadrado de $50 \mathrm{~cm}$ de lado abrangendo três linhas da cultura de trigo.

A semeadura do trigo foi realizada em 15 de maio de 2003 e as amostragens do número médio de perfilhos foram em 17 de junho e 17 de julho de 2003, determinando-se com base em amostras aleatórias de 10 plantas na unidade de análise, aos 30 e aos 60 DAS. O rendimento foi obtido com o corte e a trilha das plantas da área amostral. A massa correspondente foi obtida em balança de precisão 0,01 , sendo convertido o valor em $\mathrm{kg} 0,25 \mathrm{~m}^{-2}$ para $\mathrm{t} \mathrm{ha}^{-1}$. 


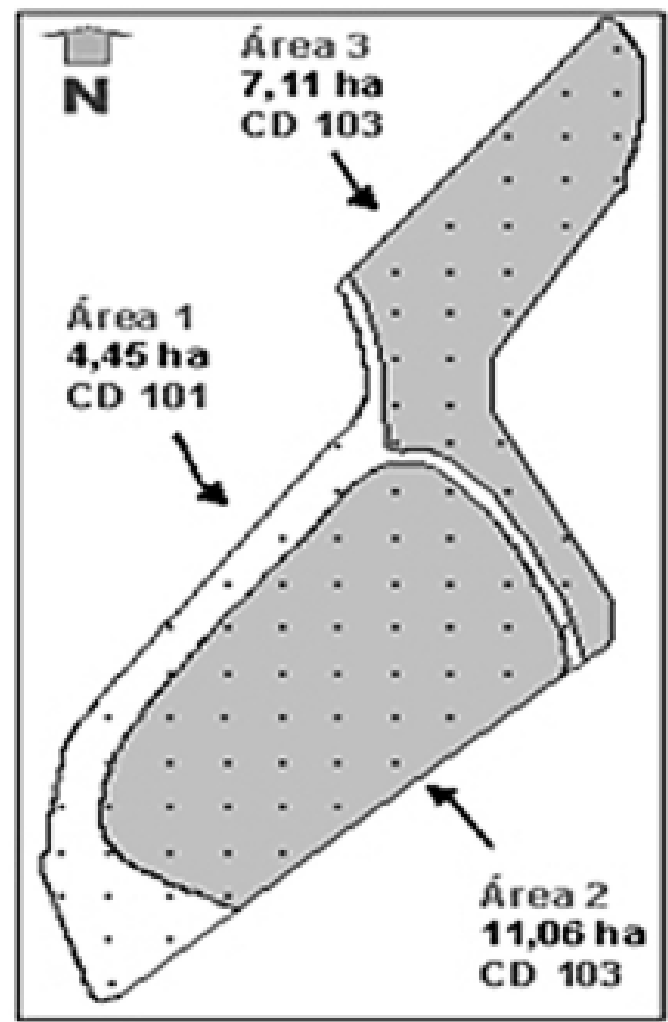

Figura 1. Esquema amostral da área do experimento, junto à Cooperativa Central de Pesquisa Agrícola. Nota: Os pontos correspondentes aos locais de amostragem foram numerados seqüencialmente da esquerda para a direita iniciando no extremo sul da área.

Para modelar um conjunto de dados com uma estrutura de correlação espacial, considera-se um processo estocástico Gaussiano $\{Z(\mathrm{~s})$,$\} em que S$ é um subconjunto de $\mathrm{R}^{d}$, sendo $d$ um espaço Euclidiano $d$ dimensional (d 1). Supondo que os $n$ dados $Z\left(s_{1}\right), \ldots, Z\left(s_{n}\right)$ do processo estocástico ou variável regionalizada $Z($.$) , são realizações conhecidas nos$ locais $s_{\mathrm{i}}, \mathrm{i}=1, \ldots, n$. em que, $s_{i}$ é um vetor $d$-dimensional. E supondo-se que os dados em forma geral podem ser escrito como $Z\left(s_{i}\right)=\left(s_{i}\right)+\left(s_{i}\right)$, sendo, $\left(s_{i}\right)$ uma função determinística que descreve a componente estrutural de $Z$ em $s_{i}$; $\left(s_{i}\right)$ é um termo estocástico, que varia localmente e depende espacialmente de $Z\left(s_{i}\right)$. Assumse que o erro estocástico $\left(s_{i}\right)$ tem média zero e a variação entre pontos no espaço é determinada pela função covariância $C\left(s_{i}, s_{j}\right)=\operatorname{Cov}\left\{\left(s_{i}\right), \quad\left(s_{j}\right)\right\}$ (Mardia e Mashall, 1984). A semivariância é uma função da distância $h$, estimada em um conjunto discreto de distâncias (lags) e está associada com a função de covariância por $=C(0)-C\left(s_{i}, s_{j}\right)$, sendo $h=$ ||$s_{i}-s_{j}||$ e C(0) a variância de Z.

Segundo IsAaKs e SRIVASTAVA (1989), a função semivariância é definida na Equação (1) como metade da esperança matemática do quadrado da diferença entre os valores de pontos no espaço, separados pelo vetor $h$ :

$$
\gamma(h)=1 / 2 E[Z(s)-Z(s+h)]^{2}
$$

em que, $Z(s)$ e $Z(s+h)$ são valores da variável regionalizada $Z$ no espaço, $d=1,2$ ou 3 , nas posições s e $s+h$, sendo $h$ a distância que separa duas amostras; $\mathrm{N}(h)$ corresponde ao número de pares de valores amostrados separados pela distância $h$. Assim, o semivariograma experimental é um gráfico versus $h$ que permite uma análise variográfica $Z(s)$ (CRESSIE, 1993).

A estrutura de dependência espacial de um processo estocástico Gaussiano $Z($.) é estudada pelo semivariograma experimental dos momentos, definida pela função semivariância da Equação 2.

$$
\hat{\gamma}(h)=\frac{1}{2 N(h)} \sum_{i=1}^{N(h)}\left[Z\left(s_{i}\right)-Z\left(s_{i}+h\right)\right]^{2}
$$

Para o caso de ter pontos discrepantes, que não possam ser eliminados ou substituídos, a bibliografia recomenda o estimador de CRESSIE e HAWKINS (1980), apresentado na Equação 3.

$$
\hat{\gamma}_{C}(h)=\frac{\left\{\frac{1}{N(h)} \sum_{i=1}^{N(h)}\left|Z\left(s_{i}\right)-Z\left(s_{i}+h\right)\right|^{\frac{1}{2}}\right\}^{4}}{0,914+\frac{0,988}{N(h)}}
$$

Verificando que o processo estocástico Z(.) seja isotrópico e intrinsecamente estacionário, a avaliação da existência de variabilidade espacial foi realizada pela técnica dos envelopes, a qual consiste em gerar intervalos para as semivariâncias estimadas ao considerar independência espacial entre as amostras (Ribeiro Junior et al., 2003; Diggle e Ribeiro Junior, 2007). Pode-se ajustar um modelo teórico ao semivariograma experimental, tais como: esférico, exponencial, circular, gaussiano, família Matérn e famílias exponencial potência, na qual se obtêm três parâmetros: alcance $(a)$ que indica a distância máxima de dependência espacial entre as amostras; o patamar $\left(\mathrm{C}=\mathrm{C}_{0}+\mathrm{C}_{1}\right)$ que é o valor máximo do semivariograma correspondente ao seu alcance onde ele se estabiliza e o efeito pepita $\left(C_{0}\right)$ indica as variações da função aleatória que não são descritas pela variabilidade espacial e as variações que podem ocorrer em distâncias menores à amostrada, com os quais, juntamente com o modelo, definem a estrutura de dependência espacial da variável em estudo. Os parâmetros da estrutura de variabilidade espacial de cada variável espacialmente referenciada foram estimados utilizando o método de máxima verossimilhança (MARDia e MASHALL, 1984). 
O grau de dependência espacial entre amostras, foi medido pelo coeficiente de efeito pepita relativo (CEPR), obtido pela Equação 4, o qual foi mencionado por CAMBARDELla et al. (1994), que classificaram a dependência espacial como forte se $C E P R<0,25$; moderada se $0,25=C E P R=0,75$ e fraca ou baixa se $C E P R>0,75$.

$$
C E P R=\frac{C_{o}}{C_{o}+C_{1}}
$$

A interpolação para locais não amostrados foi realizada por krigagem universal (CRESSIE, 1993).

Para avaliar a qualidade dos valores estimados foram utilizados os gráficos de dispersão dos valores estimados com os dados amostrados e compararamse as diferenças entre eles.

O estudo da correlação espacial entre duas variáveis regionalizadas $Z_{1}$ e $Z_{2}$ no mesmo espaço paramétrico, que possuem ocorrências nos mesmos locais, foi realizada pelo semivariograma cruzado, obtido pela Equação 5 (ISAAKs e SRIVASTAVA, 1989; CRESSIE, 1993) para processos estocásticos com estrutura de dependência espacial.

$$
\left.\hat{\gamma}_{12}(h)=\frac{1}{2 N(h)} \sum_{i=1}^{N(h)}\left\{Z_{1}\left(s_{i}+h\right)-Z_{1}\left(s_{i}\right)\right]\left[Z_{2}\left(s_{i}+h\right)-Z_{2}\left(s_{i}\right)\right]\right\}
$$

em que, $Z_{1}$ é o valor amostrado da primeira variável regionalizada e $Z_{2}$ é o valor amostrado da segunda variável regionalizada (ISAAKS e SRIVASTAVA, 1989).

Para a análise geoestatística dos dados foi utilizado o sistema estatístico R (IHAKA e GENTLEMAN, 1996) e seus módulos: geoR (RIBEIRo Junior e Diggle, 2001), Splancs (Rowlingson e Diggle, 1993) e Mass (IhaKa e Gentleman, 1996).

\section{RESULTADOS E DISCUSSÃO}

Observou-se na cultivar CD 103 (Tabela 1) maior número médio de perfilhos (NMP) por planta nos dois estádios de desenvolvimento, aos 30 (NMP30) e aos 60 DAS (NMP60), enquanto o rendimento médio (REND) da cultura foi relativamente maior para a cultivar CD 101, ainda que a dispersão do rendimento da cultura da cultivar seja maior. Também pode se verificar na Tabela 1 que existe média e alta heterogeneidade nos dados coletados, devido ao coeficiente de variação ser maior que 20\% (GOMES, 2000).

Pela análise gráfica box-plot (classificação dos dados pelos respectivos quartis), os dados apresentaram um comportamento simétrico para as duas cultivares, tanto para o número médio de perfilhos por planta aos 30 e 60 DAS, quanto para o rendimento da cultura. Ocorreram pontos discrepantes para as três variáveis regionalizadas (NMP30, NMP60 e REND) os quais não afetaram a distribuição dos dados. As diferenças que ocorreram nas estatísticas não requerem o tratamento das cultivares e mesmo das áreas como extratos. Como não houve grande variação entre as cultivares CD 101 e CD103, considerou-se para efeito de análise estatística espacial uma única variedade.

Pelos gráficos post-plot do rendimento médio da cultura e do número médio de perfilhos por planta aos 30 e 60 DAS (Figura 2) e o rendimento do trigo (Figura 2c) verificou-se tendência direcional quadrática da esquerda para a direita, isto é, na coordenada X. As parcelas com valores mais elevados estão posicionadas na região central da área. A região com os maiores resultados na amostragem 30 DAS (Figura 2a), coincide com a área em se observou os menores resultados na amostragem 60 DAS (Figura 2b).

Tabela 1. Estatísticas descritivas do rendimento da cultura de trigo e do número médio de perfilhos por planta aos 30 e 60 dias após a semeadura (DAS)

\begin{tabular}{lcccccccccc}
\hline Variáveis & Cultivar & Média & S & CV & Mín & Q1 & Me & Q3 & Máx & N \\
\hline \multirow{2}{*}{ NMP30 } & & & $\%$ & & & & & \\
& CD 101 & 1,08 & 0,29 & 27,20 & 0,60 & 0,85 & 1,10 & 1,35 & 1,50 & 19 \\
\multirow{2}{*}{ NMP60 } & CD 103 & 1,33 & 0,50 & 37,35 & 0,30 & 0,92 & 1,40 & 1,67 & 2,60 & 70 \\
& CD 101 & 1,59 & 0,70 & 43,73 & 0,50 & 1,20 & 1,60 & 2,10 & 2,80 & 19 \\
\multirow{2}{*}{ REND } & CD 103 & 1,67 & 0,62 & 37,01 & 0,40 & 1,30 & 1,70 & 2,10 & 3,40 & 70 \\
& CD 101 & 3,53 & 1,02 & 28,93 & 1,88 & 3,11 & 3,49 & 4,04 & 5,95 & 17 \\
& CD 103 & 3,33 & 0,71 & 21,42 & 1,48 & 3,02 & 3,35 & 3,66 & 5,28 & 67 \\
\hline
\end{tabular}

NMP30: número médio de perfilhos 30 DAS. NMP60: número médio de perfilhos 60 DAS. REND: rendimento da cultura. S: desvio-padrão. CV: coeficiente de variação. Mín; valor mínimo amostrado. Q1:primeiro quartil. Me: mediana. Q3: terceiro quartil. Máx: valor máximo. N: número de amostras. 
A variação gradativa da ocorrência do número médio de perfilhos aos 30 e 60 DAS caracterizou tendência direcional quadrática e foi considerada na análise de variabilidade espacial e previsão de valores para locais não amostrados.

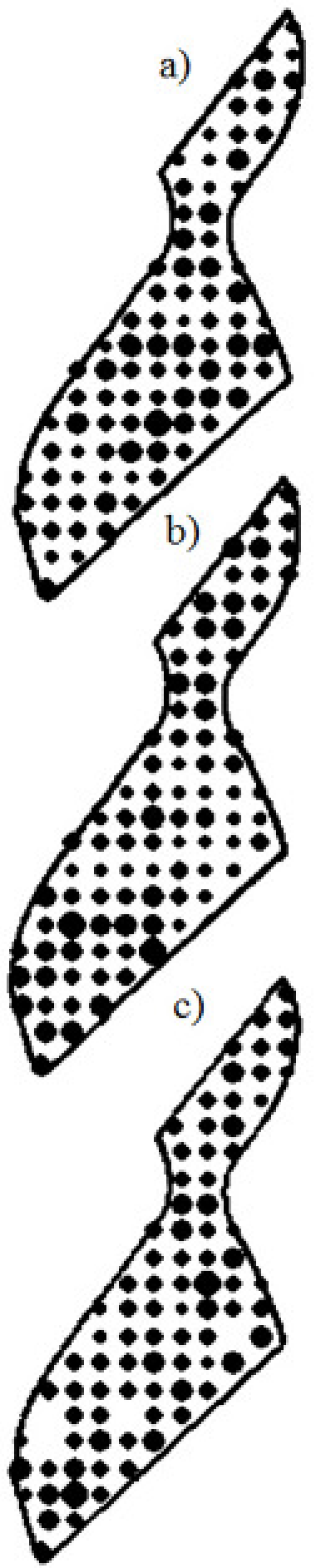

Figura 2. Classificação dos dados amostrais e das localizações de amostragem segundo os quartis a que os dados pertencem, para rendimento e número de perfilhos. Nota: o tamanho do símbolo é proporcional ao valor amostrado.
O comportamento do rendimento médio de grãos (Figura 3c) permite verificar que 9,5\% dos elementos amostrados estavam abaixo da média nacional, que em 2003 foi de $2,4 \mathrm{t} \mathrm{ha}^{-1}$ (BRAsIL, 2004) e, $90,5 \%$ das parcelas teve rendimento entre 2,5 e 5,95 t ha ${ }^{-1}$.
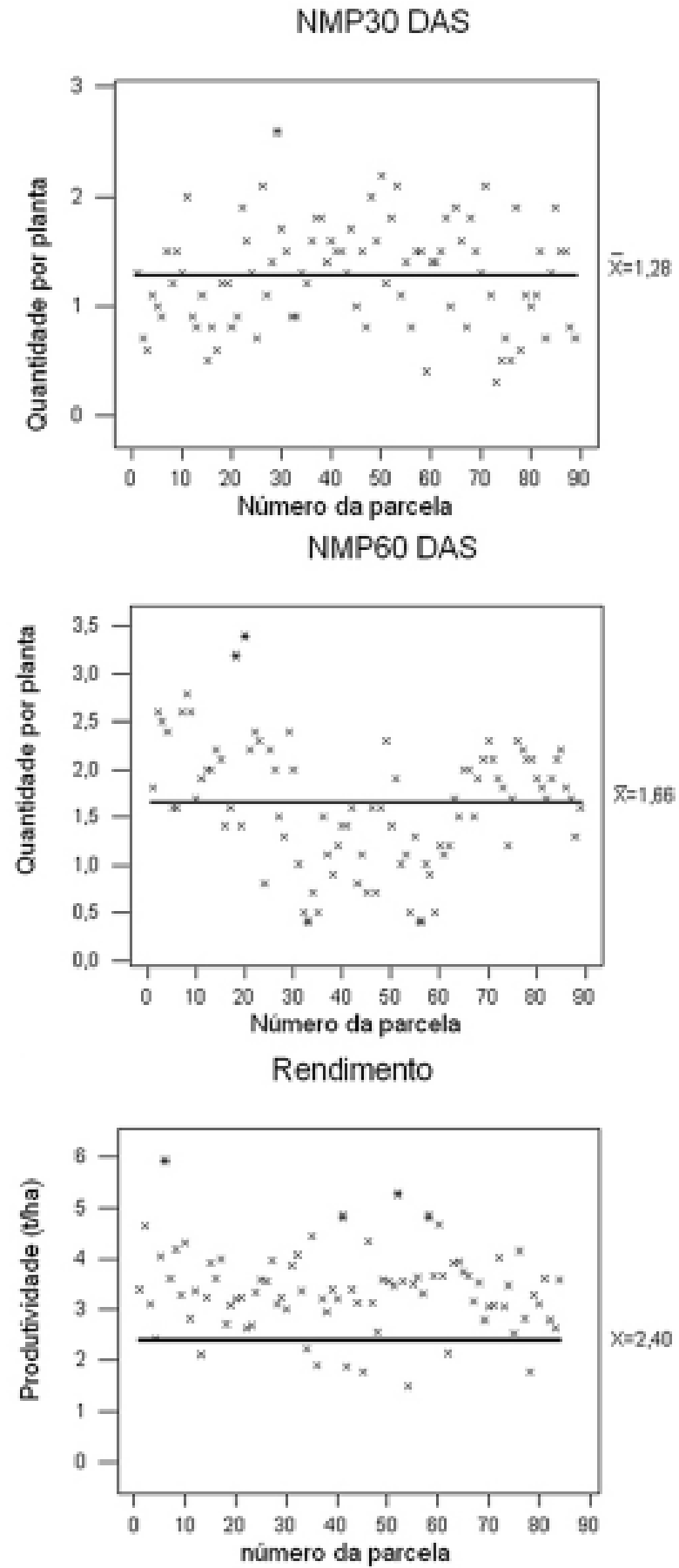

Figura 3. Comportamento do número médio de perfilhos aos 30 DAS (a) e 60 DAS (b) por planta de trigo e do rendimento da cultura (c) pelo número da parcela. Nota: A linha horizontal em (a) e (b) indica o valor médio dos dados e em (c) indica o rendimento médio da cultura no Brasil no ano agrícola 2003/2004. 
O comportamento do número médio de perfilhos por planta de trigo (Figura 3a e 3b) mostra que houve uma inversão de medidas na região central da figura, vindo a confirmar a suspeita de redução no número médio de perfilhos levantados pela análise do gráfico anterior.

$\mathrm{Na}$ amostragem aos 30 DAS verifica-se que os resultados mais elevados coincidem com as parcelas intermediárias, os quais estão acima da média obtida (reta que divide o gráfico da Figura 3). Aos 60 DAS, nessas mesmas parcelas verificaram-se os menores valores amostrados, e, como se percebe pela escala dos gráficos, não houve apenas aumento no número médio de perfilhos das demais parcelas. Nas parcelas intermediárias, a quantidade de perfilhos médios por planta diminuiu, sugerindo que as variações não foram apenas devido ao acaso ou ao tardio desenvolvimento da planta e fatores externos, como o elevado índice pluviométrico em um curto período, podem ter afetado o desenvolvimento da planta.

Os dados de precipitação pluvial da estação meteorológica próxima ao local do experimento, fornecidos pelo SIMEPAR (Sistema de Informação Metereológica do Paraná), entre a semeadura da cultura em 17/5/03 e a primeira contagem de perfilhos em 17/ 6/03 (112,5 mm de precipitação ocorrida de 3 a 6 e 9 de junho de 2003) permitem inferir que o aumento de chuvas em um curto espaço de tempo pode ter causado encharcamento do solo, provocando redução do perfilhamento, coincidindo com os resultados observados por TURNER e BEGG (1978).

No segundo mês, a precipitação pluvial de $132,6 \mathrm{~mm}$, ocorrida entre 20 de junho e 16 de julho, não provocou encharcamento do solo e pode ter favorecido o aumento do número de perfilhos emersos, coincidindo com os resultados apresentados por CunHa et al. (2001).

As variáveis NMP30, NMP60 e REND (Tabela 2) possuem variabilidade espacial explicada pelo modelo esférico, com alcance inferior a $93 \mathrm{~m}$. O menor raio de dependência espacial entre amostras (alcance) foi de 40,38 m para o NMP30 com um CEPR de 0,95, cuja classificação dada por CAMBARDELLA et al. (1994) representa fraca dependência espacial. Na variável REND constatou-se um raio de dependência espacial entre amostras de $93 \mathrm{~m}$, com um CEPR de 0,68 indicando dependência espacial moderada. Para os dados do NMP60, verificou-se um raio de dependência espacial de 91,90 m e forte dependência espacial.

Os limitantes das semivariâncias estimadas (envelopes) (Figura 4) mostraram forte dependência espacial pelos pontos fora das bandas em MNP60 e REND.

Tabela 2. Modelo teórico e parâmetros da dependência espacial do NMP30, NMP60 e REND, utilizando o método de estimação da máxima verossimilhança

\begin{tabular}{lccccc}
\hline Variável & $\begin{array}{c}\text { Modelo } \\
\left(\mathrm{C}_{0}\right)\end{array}$ & $\begin{array}{c}\text { Efeito Pepita } \\
\left(\mathrm{C}=\mathrm{C}_{0}+\mathrm{C}_{1}\right)\end{array}$ & $\begin{array}{c}\text { Patamar } \\
(a)\end{array}$ & $\begin{array}{c}\text { Alcance } \\
\mathrm{C}_{0} /\left(\mathrm{C}_{0}+\mathrm{C}_{1}\right)\end{array}$ & CEPR \\
\hline NMP30 & Esférico & 0,18 & 0,19 & 40,38 & 0,95 \\
NMP60 & Esférico & 0,00 & 0,30 & 91,83 & 0,00 \\
REND & Esférico & 0,40 & 0,58 & 93,00 & 0,68 \\
\hline
\end{tabular}

NMP30: Número médio de perfilhos 30 dias após semeadura (DAS). NMP60: Número médio de perfilhos 60 DAS. REND: Rendimento do trigo $\left(\mathrm{t} \mathrm{ha}{ }^{-1}\right)$; CEPR: coeficiente de efeito pepita relativo.

Pela figura $4 a$, observa-se que não houve influência da localização dos pontos na estimação das semivariâncias para NMP30. A dependência espacial considerada como baixa (Tabela 2), não foi identificada pela técnica dos envelopes. Na estimação das semivariâncias para NMP60 a importância de considerar a localização dos pontos amostrados no estudo desta variabilidade foi mais evidente. $\mathrm{Na}$ figura $4 \mathrm{~b}$ verifica-se que duas semivariâncias extrapolaram os limites dos envelopes que definem a região de independência espacial. Para o REND (Figura 4c) a variabilidade ocorrida nos valores amostrados não foi provocada apenas por fatores aleatórios, uma semivariância extrapolou os limites gerados que definem a região de independência espacial, mas ficou bem próximo ao limite.

Nas figuras $5 a, 5 b$ e 5 c são apresentados os mapas de contorno do número médio de perfilhos aos 30 e 60 DAS e do rendimento da cultura, respectivamente, construídos utilizando a interpolação por krigagem ordinária e a estrutura de variabilidade espacial indicada na tabela 2.

Nos mapas temáticos de contorno do número médio de perfilhos, NMP30 e NMP60 (Figuras 5a e $5 b$ ) respectivamente, a diferença primordial foi nos detalhes em que o mapa de NMP60 representou em contraste com as regiões bem definidas de valores de 
perfilhamento indicadas em NMP30 (Figura 5a). Houve pouca variação na área, tendo em média dois perfilhos por planta. Já em NMP60 houve aumento de perfilhos nas regiões extremas e na região central, menor quantidade de perfilhos. No mapa temático do rendimento do trigo (Figura 5c) notaram-se setores bem definidos. Comparando os resultados com a média brasileira de rendimento do trigo em 2003, percebem-se (Figura $5 \mathrm{c}$ ), em toda a área, valores superiores à média nacional $\left(2,4 \mathrm{t} \mathrm{ha}^{-1}\right)$, variando de 2,5 a $4,5 \mathrm{t} \mathrm{ha}^{-1}$.
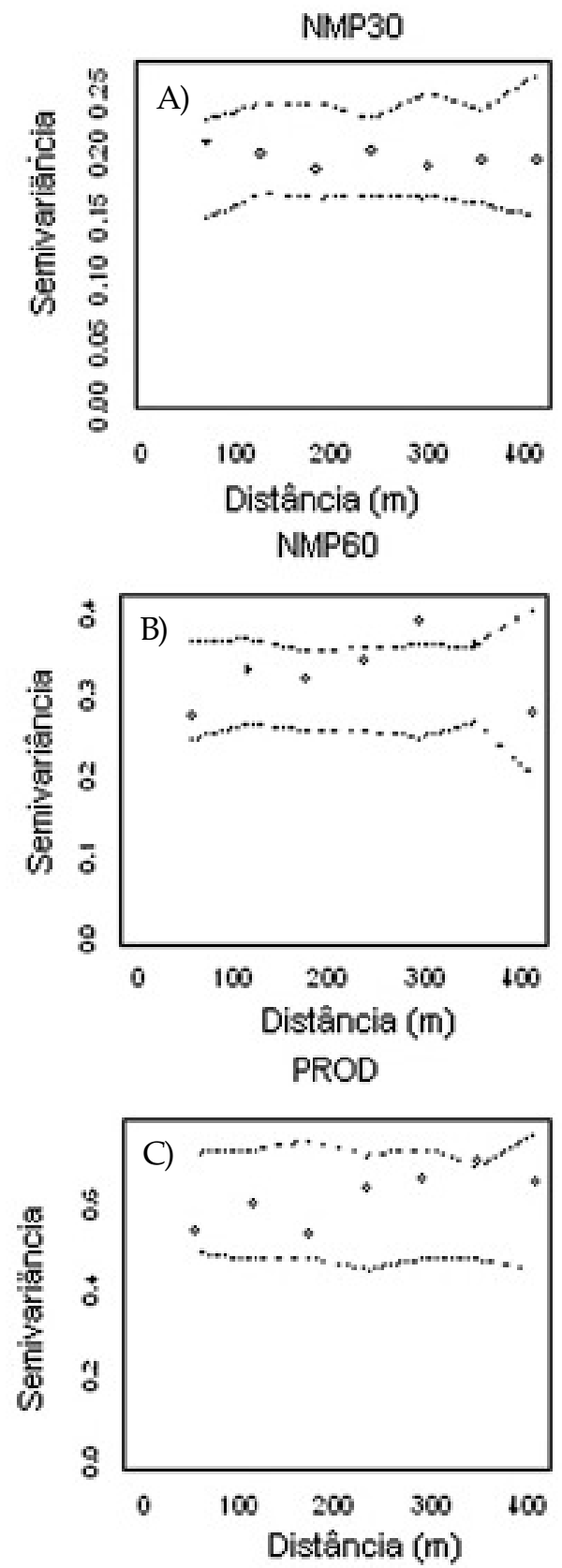

Figura 4. Limitantes das semivariâncias, envelopes, gerados desconsiderando a localização amostral.A) número médio de perfilhos aos 30 DAS; B) número médio de perfilhos aos 60 DAS; C) rendimento do trigo em $t h^{-1}$.

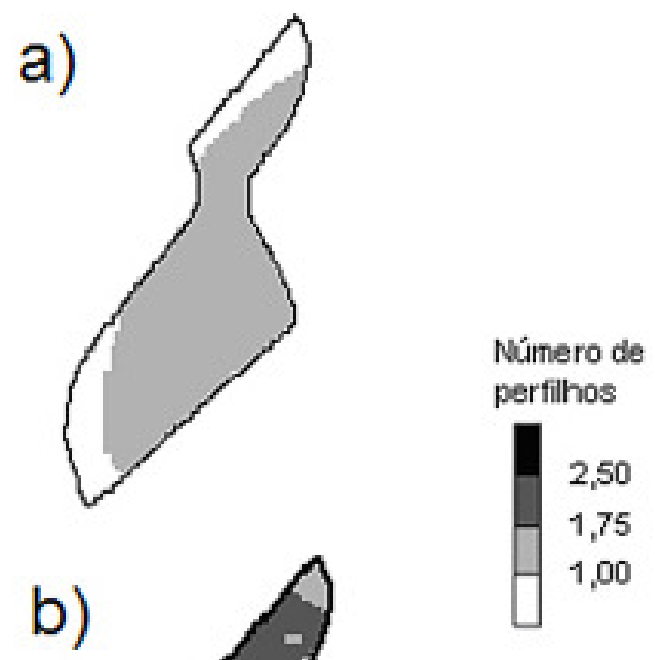

PROD

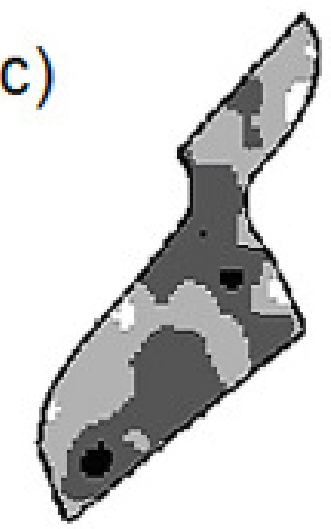

4,50
4,00
3,50
3,00
2,50

Figura 5. Mapas temáticos do número médio de perfilhos aos 30 (A) e 60 DAS (B) e do rendimento do trigo (C), interpolados por krigagem.

Com as evidências na interpolação do rendimento (Figura 6), verifica-se que a média amostral foi mais valorizada que os valores amostrais. No eixo dos valores estimados os dados estão concentrados entre os valores 3 e $4 \mathrm{tha}^{-1}$. 
Para os dados do número médio de perfilhos houve maior dispersão dos valores estimados, sendo maior a dispersão quando se tratou do NMP60 (Figura $6 \mathrm{~b})$ indicando ser os valores amostrados e a localização espacial da amostragem mais intensamente considerados do que a média amostral. Esse fato é decorrente de os dados de NMP60 indicarem forte dependência espacial e os dados de NMP30 e REND, dependência espacial baixa e moderada respectivamente.
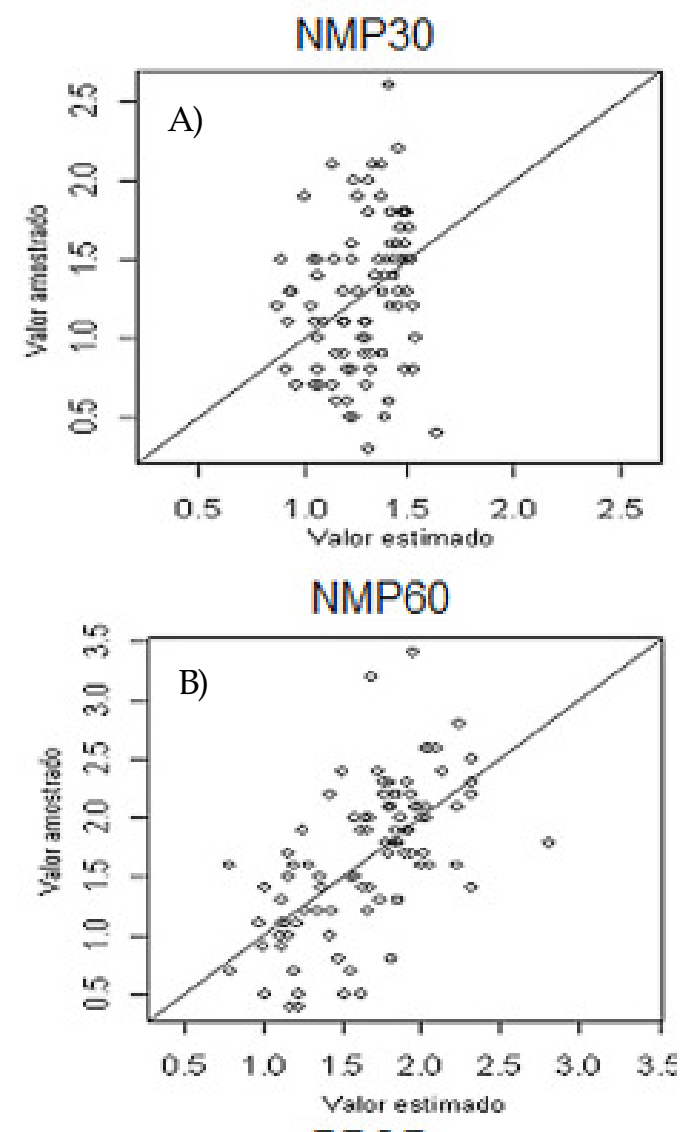

PROD

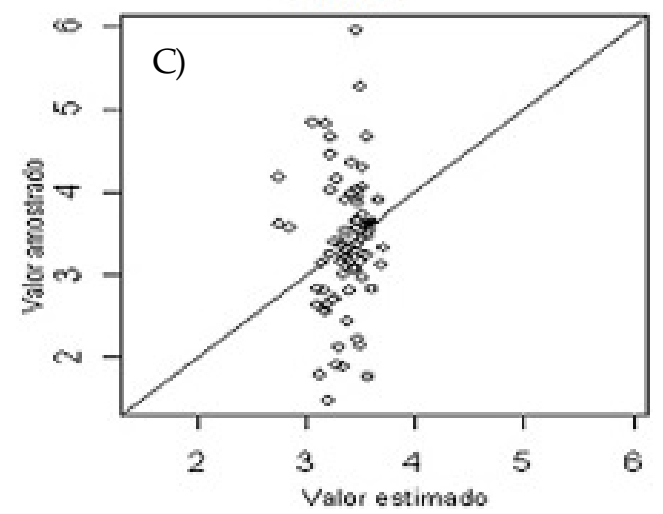

Figura 6. Dispersão dos valores amostrados pelos valores preditos por krigagem do rendimento de trigo e do número médio de perfilhos por planta. A) número médio de perfilhos aos 30 DAS; B) número médio de perfilhos aos 60 DAS; C) rendimento do trigo em $\mathrm{t} \mathrm{ha}^{-1}$.
Pela comparação dos valores estimados e amostrados em seus locais de ocorrência, indicando as parcelas que foram superestimadas e subestimadas (Figura 7), observa-se em NMP30, menor percentual de parcelas superestimadas ( $54 \%$ para as demais variáveis (Figura $7 \mathrm{~b}$ e $7 \mathrm{c}$ ). Para os três conjuntos de dados, a quantidade de parcelas superestimadas e subestimadas foi semelhante, indicando bom ajuste e confirmando as características do interpolador por krigagem de ser bom estimador não viciado e com variância mínima (BLUE).
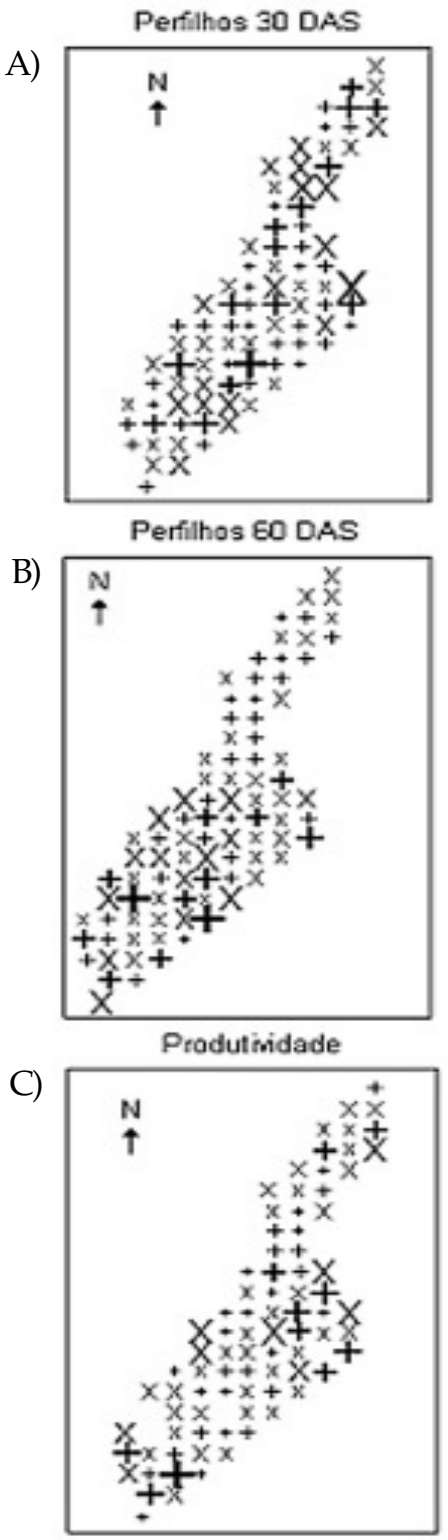

Figura 7. Comparação localizada na área entre valores amostrados e estimados por krigagem para a produtividade do trigo e o número médio de perfilhos por planta. A) número médio de perfilhos aos 30 DAS; B) número médio de perfilhos aos 60 DAS; C) rendimento do trigo em $\mathrm{t} \mathrm{ha}^{-1}$. Nota: $\mathrm{O}$ tamanho dos símbolos é proporcional aos valores que correspondem. 
Os símbolos proporcionais à diferença existente entre os valores estimados e os amostrados permitem verificar que as estimativas feitas para NMP60 distam menos dos valores amostrados se comparados com NMP30. Para REND (Figura 7c), em várias parcelas notaram-se valores estimados e amostrados com diferenças pequenas, tanto para valores superestimados como subestimados, o que pode ser comprovado pelo acúmulo de pontos junto à reta de calibração (Figura $6 \mathrm{c}$ ).

Pelo índice de correlação espacial (Tabela 3), utilizando o semivariograma cruzado e o coeficiente de correlação espacial constatado por WACKERNAGEL (1999) das variáveis NMP30, NMP60 e REND, percebese que o maior índice de correlação espacial do REND foi com NMP30. Confirmando a evidência revelada pelos mapas temáticos, a correlação espacial dada pelo semivariograma cruzado entre NMP30 e NMP60 foi de $-0,29$, mostrando correlação inversa e mais forte entre NMP30 e NMP60 e NMP60 e REND de 0,10, comprovado pelas análises do gráfico postplot (Figura $2 a$ e $2 b$ ) e da dispersão dos dados segundo os locais de amostragem (Figura 3a e 3b).

Tabela 3. Índices de correlação entre dados de perfilhos aos 30 e 60 DAS e o rendimento do trigo

\begin{tabular}{lccc}
\hline Variáveis & NMP30 & NMP60 & REND \\
\hline NMP30 & 1,00 & - & - \\
NMP60 & $-0,29$ & 1,00 & - \\
REND & 0,21 & 0,10 & 1,00 \\
\hline
\end{tabular}

NMP30: Número médio de perfilhos 30 dias após semeadura (DAS). NMP60: Número médio de perfilhos 60 DAS. REND: Rendimento do trigo $\left(\mathrm{t} \mathrm{ha}^{-1}\right)$.

\section{CONCLUSÕES}

1. Pelas amostragens realizadas no estudo e pelo estudo geoestatístico foi possível obter a estrutura de dependência espacial para as variáveis estudadas.

2. Por meio do semivariograma cruzado verificou-se a existência de correlação espacial, principalmente entre o rendimento do trigo e o número médio de perfilhos aos 30 dias após a semeadura.

3. Os mapas gerados permitiram melhor visualização espacial de cada variável regionalizada, facilitando, assim, o gerenciamento da propriedade.

\section{AGRADECIMENTOS}

Ao CNPq, à CAPES e à Fundação Araucária pelo apoio financeiro, e à COODETEC pelo apoio técnico.

\section{REFERÊNCIAS}

ALVES, A.C., MUNDSTOCK, C.M. MEDEIROS, J.D. Sistema vascular e controle do desenvolvimento de perfilhos em cereais de estação fria. Revista Brasileira de Botânica, São Paulo, v. 23, n. 1, p. 59-67, 2000.

BRASIL, Ministério de Agricultura, Pecuária e Abastecimento. Informações técnicas das comissões centro-sul brasileiras de pesquisas de trigo e de triticale para a safra de 2004. Londrina: Embrapa, 2004. 218p.

CAMBARDELLA, C.A.; MOORMAN, T.B.; NOVAK, J.M.; PARKIN, T.B.; KARLEN, D.L.; TURCO, R.F.; KONOPKA, A.E. Field-scale variability of soil properties in Central Iowa Soils. Soil Science Society America Journal, Madison, v.58, n. 5, p.1501-1511, 1994.

CRESSIE, N. Statistics for spatial data. New York: Jonh Wiley, 1993. 900p.

CRESSIE, N.; HAWKINS, D.M. Robust estimation of the variogram, I. Mathematical Geology, New York, v. 12, n. 2, p. $115-125,1980$.

CUNHA, M.A.D; LEITE, G.G.; DIOGO, J.M.S.; VIVALDI, L. Características morfológicas do Paspalum atratum cv. pojuca submetido ao pastejo rotacionado. Dinâmica de perfilhamento e elongação de folhas. Revista Brasileira de Zootecnia, Viçosa, v. 30, n. 3, p. 935-940, 2001.

DIGGLE, P.J.; RIBEIROJUNIOR, P.J. Model based geoestatistics. New York: Springer Series in Statistics, USA, 2007. 228p.

EMBRAPA - Serviço Nacional de Levantamento e Conservação de Solos. Sistema Brasileiro de Classificação de solos. Brasília: Embrapa Produções de Informações, 1999, 412p.

GOMES, F.P. Curso de estatística experimental. 14 ed. Piracicaba: Nobel, 2000. 477p.

IHAKA, R.; GENTLEMAN, R.A language for data analysis and graphics. Journal of Computational and Graphical Statistics, Salt Like City, v. 5, n. 3, 1996. p. 229-314. Software disponível em: http://www.R-project.org. Acesso em 31/8/2003.

ISAAKS, E.H.; SRIVASTAVA, R.M. An introducion to applied geostatistic. New York: Oxford University Press, 1989. 561p.

MARDIA, K.V.; MARSHALL, R.J. Maximum likelihood models for residual covariance in special regression. Biometrika, Great Britain. v. 71, n. 1, p. 135-146, 1984.

MATHERON, G. Principles of geostatistics. Economic Geology, Lancaster, v. 58, n. 8, p. 1246-1266, 1963.

RIBEIRO JUNIOR, P.J; DIGGLE, P.J. geoR: A package from geoestatistical analysis. R_NEWS. University Lancaster, UK, v. 1, n. 2, 2001. p. 15-18. Disponível em: http:// cran.R-project.org/doc/Rnews. Acesso em 31/10/2003. 
RIBEIRO JUNIOR, P.J; CHRISTENSEN, O.F; DIGGLE, P.J.Geostatistical software - geoR and geoRglm. Disponível em: www.est.ufpr.br/ce714. Acesso em: 12/10/2003.

ROWLINGSON, B.; DIGGLE, P.J. Splancs: spatial point pattern analysis code in S-Plus. Computers and Geosciences, Canada, v. 19 , n. 5, p. 627-655, 1993.

TAMAS, I.A. Hormonal regulation of apical dominance. In: DAVIES, P. J. (Ed.). Plant hormones: physiology, biochemistry and molecular biology. 2.ed. Dordrecht: Kluwer Academic, 1995. cap. G6, p. 572-597.
TONET, G.L. Resistência de plantas de trigo ao pulgão verde dos cereais. Passo Fundo: Embrapa Trigo, 1999. 3p. html. (Embrapa Trigo. Comunicado Técnico Online, 17). Disponível em: www.cnpt.embrapa.br/biblio/p_co17.htm. Acesso em $31 / 8 / 2006$.

TUNEER, N.C.; BEGG, J.E. Response of pastre plants to water déficits. In: WILSON, J. R. (Ed.). Plant relations in pasture. Melbourne: CSIRO; p. 50-66. 1978.

WACKERNAGEL, H. Multivariate geoestatistics. 2.ed. New York: Springer, 1999. 291p. 